\title{
ESTUDO SOBRE O SOFTWARE DE MAPEAMENTO OBJETO-RELACIONAL PEEWEE
}

\author{
Luís Fernando Alves da Silva \\ Pedro Ramires da Silva Amalfi Costa \\ Reginaldo Donizeti Cândido \\ Mogi Guaçu SP
}

DOI:

\section{RESUMO}

Este artigo científico tem como objetivo explanar a do software de função do software de mapeamento objeto-relacional Peewee, partindo do conceito dos sistemas gerenciadores de banco de dados a linguagem SQL, seguindo pelos banco de dados suportados por ele (MySQL, PostgreSQL e SQLite), até a apresentação do conceito de ORM e algumas especificardes do Peewee, como criar o modelo, sincronizar, popular as tabelas e finalizando com as operações do CRUD (criar, ler atualizar e remover dados).

Palavras-chave: Peewee; ORM; SGBD; SQL; CRUD. 


\title{
1 INTRODUÇÃO
}

A persistência de dados é um conceito fundamental para a maioria das aplicações modernas.

\begin{abstract}
Em ciência da computação, persistência se refere à característica de um estado que sobrevive ao processo que o criou. Sem essa capacidade, o estado só existiria na RAM, e seria perdido quando a RAM parasse (desligando-se 0 computador por exemplo). (CALLIGARO, 2005)
\end{abstract}

Entre as diversas formas possíveis para persistir dados em sistemas computacionais os Sistemas Gerenciadores de Banco de Dados (SGBD) se destacam.

\begin{abstract}
Um Sistema de Gerenciamento de Banco de Dados (SGBD) - do inglês Data Base Management System (DBMS) - é o conjunto de softwares responsáveis pelo gerenciamento de um banco de dados. Seu principal objetivo é retirar da aplicação cliente a responsabilidade de gerenciar o acesso, a persistência, a manipulação e a organização dos dados. (ALVES, 2013)
\end{abstract}

Embora possamos armazenar e recuperar informações de forma simples sem recorrermos a SGBDs o uso deles se tornou padrão para a maioria das tarefas que envolvem a persistência de dados, sendo considerados uma solução robusta e confiável para esta tarefa.

Entretanto os Banco de Dados são sistemas complexos compostos de diversos subsistemas e a forma pela qual interagimos com eles envolve 0 conhecimento de diversas tecnologias, como a linguagem de consulta SQL.

O SGBD disponibiliza uma interface para que seus clientes possam incluir, alterar ou consultar dados previamente armazenados. Em bancos de dados relacionais a interface é constituída pelas APIs (Application Programming Interface) ou drivers do SGBD, que executam comandos na linguagem SQL (Structured Query Language). (ALVES, 2013)

Apesar de ter sido criada como um padrão da indústria para permitir a estruturação de uma consulta ao banco de dados a SQL acabou se tornando 
levemente diferente a cada implementação pois cada fabricante de SGBD criou pequenas variações de sintaxe e de instruções para diferenciar o seu produto.

Esse cenário acabou criando uma fragmentação tecnológica, onde para cada SGBD o programador deve conhecer as peculiaridades da implementação da SQL utilizada por ele, isso faz com que migrações de banco de dados sejam tarefas custosas.

Outro fator que dificulta a utilização de SGBDs é que o funcionamento dos Banco de Dados Relacionais e da linguagem SQL, difere significativamente das linguagens de programação, esse fator dificulta a sua utilização principalmente por programadores iniciantes.

Nesse contexto surgem os softwares de Mapeamento Objeto-Relacional também conhecidos pela sigla em inglês ORM.

ORM (Object Relational Mapper) é uma técnica de mapeamento objeto relacional que permite fazer uma relação dos objetos com os dados que os mesmos representam. Ultimamente tem sido muito utilizada e vem crescendo bastante nos últimos anos. Este crescimento tem se dado principalmente pelo fato de muitos desenvolvedores não se sentirem a vontade em escrever código SQL e pela produtividade que esta técnica nos proporciona. (DEVMEDIA, 2019)

Existem diversos softwares ORM, e estes variam de acordo com a linguagem de programação utilizada e com quais SGBDs eles são capazes de se comunicar.

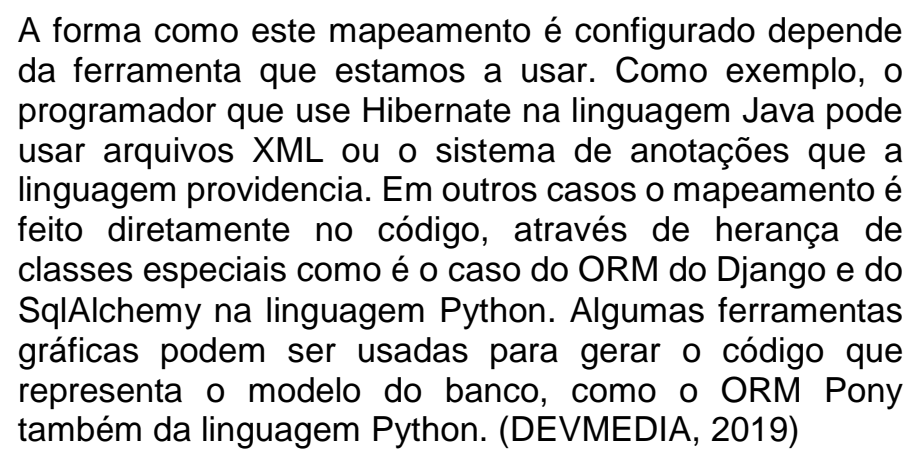

Este artigo vai apresentar o software ORM Peewee, implementado com a linguagem de programação Python. 


\title{
2 SISTEMAS GERENCIADORES DE BANCO DE DADOS (SGBD)
}

Neste capítulo será abordado de forma resumida o surgimento dos Sistemas Gerenciadores de Banco de Dados (SGBD), seguido por uma introdução a linguagem SQL assim como alguns dos principais SGBDs disponíveis no mercado.

\subsection{O SURGIMENTO DOS SISTEMAS GERENCIADORES DE BANCO DE DADOS (SGBD) RELACIONAIS}

Os Sistemas Gerenciadores de Banco De Dados sugiram durante os anos 60 , a principal motivação foi a busca por maneiras mais rápidas e baratas de se gerenciar grandes quantidades de informação.

\begin{abstract}
Igualmente a muitas tecnologias na computação industrial, os fundamentos de bancos de dados relacionais surgiram na empresa IBM, nas décadas de 1960 e 1970, através de pesquisas de funções de automação de escritório. Foi durante um período da história na qual empresas descobriram que estava muito custoso empregar um número grande de pessoas para fazer trabalhos como armazenar e indexar (organizar) arquivos. Por este motivo, valia a pena os esforços e investimentos em pesquisar um meio mais barato e ter uma solução mecânica eficiente. (PROFISSAODBA, 2019)
\end{abstract}

Diversos modelos de SGBDs foram desenvolvidos, entretanto um modelo se tornou o mais popular nas décadas seguinte, o modelo relacional. Nesse sistema os dados são organizados em tabelas denominadas entidades, cada tabela possui uma estrutura composta de campos com tipos específicos de dados, alguns desses campos podem conter chaves que permitem relacionar os dados de diversas tabelas.

Em 1970 um pesquisador da IBM - Ted Codd - publicou o primeiro artigo sobre bancos de dados relacionais. Este artigo tratava sobre o uso de cálculo e álgebra relacional para permitir que usuários não técnicos armazenassem e recuperassem grande quantidade de informações. Codd visionava um sistema onde o usuário seria capaz de acessar as informações através de comandos em inglês, onde as informações estariam armazenadas em tabelas. (PROFISSAODBA, 2019) 
Para que o Banco de Dados Relacional seja eficaz, devemos seguir algumas regras de modelagem que garantem a integridade do sistema, chamamos essas regras de normalização.

"Normalização de dados é o processo formal e passo a passo que examina os atributos de uma entidade, com o objetivo de evitar anomalias observadas na inclusão, exclusão e alteração de registros" (PROFESSOR DIGITAL, 2019).

\subsection{LINGUAGEM DE CONSULTA ESTRUTURADA (SQL)}

A manipulação dos SGBDs relacionais é feita através da Linguagem de Consulta Estruturada (SQL). Ela fornece uma interface através do qual utilizando de uma linguagem de pesquisa declarativa podemos estruturar um esquema de banco de dados. Nele é possível executar pesquisas, inserir, alterar e apagar dados além de operações mais complexas como transações.

A "Linguagem Estruturada de Consultas" (SQL, traduzida para o português) é utilizada para interagir com o SGBD e executar várias tarefas como inserir e alterar registros, criar objetos no banco de dados, gerenciar usuário, consultar informações, controlar transações, etc. Todas as operações realizadas no banco de dados podem ser solicitadas ao SGBD utilizando esta linguagem. (ALVES, 2013)

\subsection{MYSQL}

O MySQL é o SGBD mais popular do mercado, criado na Suécia em 1995 ele ganhou notoriedade por ter sido lançado como software livre e de uso gratuito.

O MySQL foi criado na Suécia por suecos e um finlandês: David Axmark, Allan Larsson e Michael "Monty" Widenius, que têm trabalhado juntos desde a década de 1980. Hoje seu desenvolvimento e manutenção empregam aproximadamente 400 profissionais no mundo inteiro, e mais de mil contribuem testando o software, integrando-o a outros produtos, e escrevendo a respeito dele. (ZANZARINI, 2019) 
Outro fator que colaborou com a difusão do MySQL foi a sua associação com a linguagem de programação PHP que junto do sistema operacional Linux e do servidor Web Apache (todas tecnologias open source e gratuitas na maioria dos usos) formam a pilha de softwares mais popular para o desenvolvimento Web conhecida como LAMP.

O MySQL é um Banco de Dados de médio porte, capaz de lidar com aplicações em redes e é o principal SGBD utilizado na Web.

Em 2008 o MySQL foi comprado pela Sun Microsystems criadora da linguagem de programação Java e posteriormente essa foi comprada pela gigante dos Banco de Dados Oracle, esse fator fez com que parte da base de desenvolvedores do MySQL criasse um fork (derivação) do projeto original chamado MariaDB.

\subsection{POSTGRESQL}

O PostgreSQL se originou em 1973 de estudos com o projeto Ingres, desenvolvido na Universidade de Berkeley, Califórnia, entretanto somente em 1996 o projeto veio a assumir o nome PostgreSQL.

O "pontapé" inicial aconteceu na universidade de Berkeley
na Califórnia (1973), quando Michael Stonebraker e
Eugene Wong, decidem iniciar um projeto de Banco de
Dados Relacional e desenvolvem o Ingres. Após isso,
Stonebraker sai do projeto para comercializar o Ingres,
mas volta logo em seguida iniciando o projeto do post-
Ingres, que buscava resolver o que considerava ser
limitações do modelo relacional. Dessa forma, o post-
Ingres começou a ganhar características de um SGBD
estendido ou objeto-relacional. Posteriormente, o nome
do sistema passou de post-Ingres (após o Ingres) para
postgres. O postgres teve entre os seus financiadores
organizações como a DARPA (Agência de Pesquisas em
Projetos Avançados), e o ARO (Escritório de Pesquisas
do Exército). (DEVMEDIA, 2019)

Uma das motivações para a criação do PostgreSQL foi a resolução de alguns problemas do modelo relacional, gerando um novo modelo chamado de SGBDRe ou também de objeto-relacional. 
Ele é um Banco de Dados de grande porte, capaz de lidar com grande quantidade de dados e acessos simultâneos, e seu uso vem crescendo a cada dia como uma alternativa open source e gratuita ao Oracle.

\subsection{SQLITE}

O SQLite se originou em 2000 no como um projeto da General para a criação de um Banco de Dados embarcado par um sistema de misseis da marinha dos Estados Unidos. Diferentemente da maioria dos SGBDs o SQLite não precisa de um servidor dedicado.

SQLite é uma biblioteca em linguagem $\mathrm{C}$ que implementa um banco de dados SQL embutido. Programas que usam a biblioteca SQLite podem ter acesso a banco de dados SQL sem executar um processo SGBD separado. SQLite não é uma biblioteca cliente usada para conectar com um grande servidor de banco de dados, mas sim o próprio servidor. A biblioteca SQLite lê e escreve diretamente no arquivo de banco de dados no disco. (OLIVEIRA, 2011)

No mesmo ano de 2000 ele foi liberado como um software open source e gratuito.

O SQLite pode ser classificado como um Banco de Dados de pequeno porte, mas com a praticidade de um software embarcado ele é utilizado em diversos projetos como nos sistemas operacionais IOS e Android além dos navegadores Google Chrome e Mozilla Firefox. 


\section{O SOFTWARE DE MAPEAMENTO OBJETO-RELACIONAL PEEWEE}

Este capítulo abordará qual é o princípio de funcionamento de um software de Mapeamento Objeto-Relacional (ORM) especificamente o Peewee.

\subsection{MAPEAMENTO OBJETO-RELACIONAL (ORM)}

Para ao integrar um SGBD relacional a um sistema o programador deve ter domínio em modelagem de dados além de possuir conhecimento da linguagem SQL. Entretanto como dito anteriormente esses conhecimentos fogem do escopo das linguagens de programação que se focam em sua maioria no uso do paradigma da programação orientada a objetos.

Mapeamento objeto-relacional (ou ORM, do inglês: Object-relational mapping) é uma técnica de desenvolvimento utilizada para reduzir a impedância da programação orientada aos objetos utilizando bancos de dados relacionais. As tabelas do banco de dados são representadas através de classes e os registros de cada tabela são representados como instâncias das classes correspondentes. Com esta técnica, o programador não precisa se preocupar com os comandos em linguagem SQL; ele irá usar uma interface de programação simples que faz todo o trabalho de persistência. Não é necessária uma correspondência direta entre as tabelas de dados e as classes do programa. A relação entre as tabelas onde originam os dados e o objeto que os disponibiliza é configurada pelo programador, isolando o código do programa das alterações à organização dos dados nas tabelas do banco de dados. (DEVMEDIA, 2011) 
Figura 1 - Camadas de um ORM

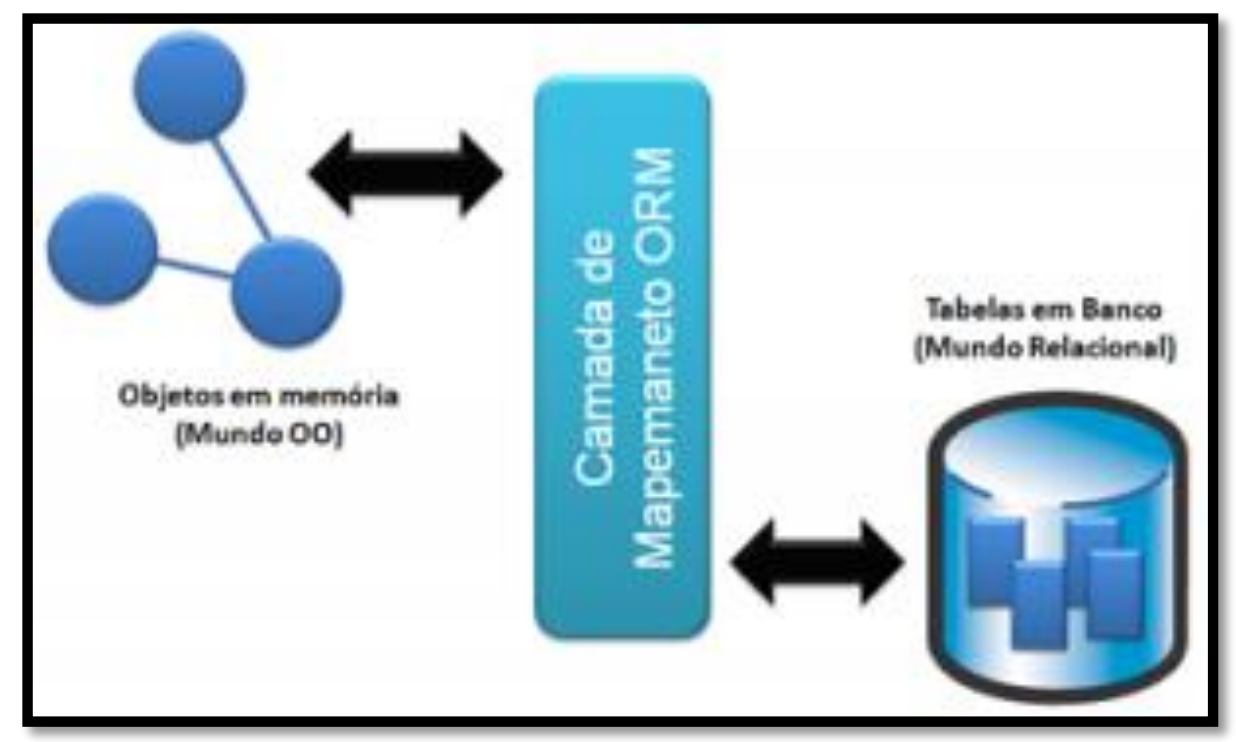

Fonte: DEVMEDIA (2019)

Nesse contexto surgem os softwares Mapeamento Objeto-Relacional, com ele os programadores fazem a modelagem através da definição de classes e o ORM mapeia essas classes em comandos SQL que são enviados ao Banco de Dados.

No mundo relacional prevalecem princípios matemáticos com a finalidade de armazenar e gerenciar corretamente os dados, de forma segura e se trabalha com a linguagem SQL que é utilizada para dizer o banco de dados "O QUE?" fazer e não como fazer. Já no mundo orientado a objetos trabalhamos com classes e métodos, ou seja, trabalhamos fundamentados na engenharia de software e seus princípios que nos dizem "COMO" fazer. O ORM é justamente, a ponte entre estes dois mundos, ou seja, é ele quem vai permitir que você armazene os seus objetos no banco de dados. (DEVMEDIA, 2019)

Além Disso o ORM gera métodos responsáveis pela interação entre o software e o Banco de Dados, esses métodos são os mesmos independente SGBD utilizado, oque torna o procedimento de migração simples.

... nos traz uma ideia de como o ORM trabalha. Ele faz o mapeamento da sua classe para o banco de dados e cada ORM tem suas particularidades para gerar o SQL referente a inserção do objeto que corresponde a uma tabela no banco de dados e realizar a operação. Utilizando um ORM, também se ganha produtividade, pois deixa-se de escrever os comandos SQL para deixar que o próprio ORM, faça isto por você. (DEVMEDIA, 2019) 
Figura 2 - Como o ORM trabalha

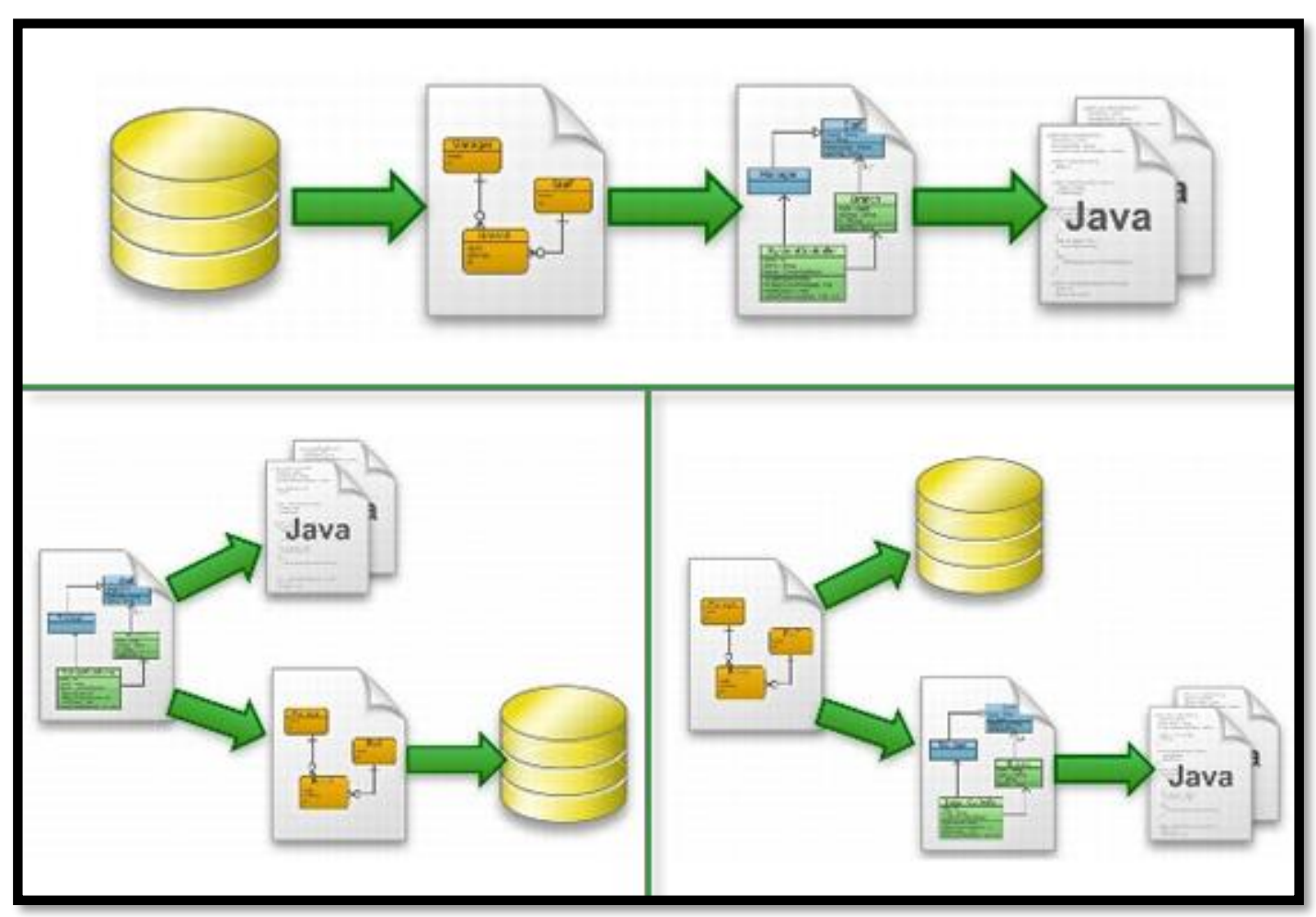

Fonte: DEVMEDIA (2019)

\subsection{O ORM PEEWEE}

O Peewee é um ORM para a linguagem de programação Python, ele foi construído com o objetivo de ser simples e pequeno, sendo de menor complexidade do que outros ORMs em Python como o SQLAlchemy.

O Peewee é destinado a projetos de pequeno/médio porte, se destacando pela simplicidade quando comparado a outros ORM mais conhecidos, como o SQLAlchemy. Uma analogia utilizada pelo autor da API e que acho muito interessante é que Peewee está para o SQLAlchemy assim como SQLite está para o PostgreSQL. (STUTTGART, 2017) 
Mesmo sendo simples ele ainda possui suporte aos Bancos de Dados SQLite, MySQL e PostgreSQL cobrindo assim os principais SGBDs relacionais open source do mercado.

Em relação aos recursos por ele oferecidos, podemos citar que ele possui suporte nativo a SQLite, PostgreSQL e MySQL, embora seja necessário a instalação de drivers para utilizá-lo com PostgreSQL e MySQL e suporta tanto Python 2.6+ quanto Python 3.4+.. (STUTTGART, 2017)

O Peewee pode ser facilmente instalado ctravez do gerenciador de pacotes do Python o pip com o comando pip install peewee.

\subsection{CRIANDO UM MODELO COM O PEEWEE}

Como na maioria dos ORM's o procedimento para se criar um modelo para ser utilizado no Peewee começa com a definição de uma classe em Python como pode ser visto na imagem a seguir.

Figura 3 - Criando um modelo no Peewee

from peewee import *

$d b=$ SqliteDatabase('pessoa.db')

class Pessoa (Model):

nome $=$ CharField ()

sobrenome $=$ CharField ()

data nascimento $=$ DateField ()

class Meta:

database $\boldsymbol{=} \mathrm{db}$

Fonte: SILVA A, COSTA A S e CÂNDIDO D (2019)

Iniciamos o nosso projeto importando toda biblioteca Peewee. 
Em seguida definimos qual o Banco de Dados iremos utilizar, neste caso o SQLite, uma observação importante e que para mudar o SGBD que a aplicação utiliza, basta alterar o método de conexão na variável db. Deve se salientar que cada Banco de Dados possui suas próprias peculiaridades e a definição do objeto db varia conforme o SGBD utilizado. Essa configuração equivale a criação de um Banco de Dados em um SGBD (CREATE DATABASE).

Após essas configurações iniciais nos partimos para a criação das entidades (tabelas) modelando as suas classes, esse procedimento é o equivalente ao comando do SQL CREATE TABLE. Para isso nos utilizamos a herança da orientação a objeto através da classe do Peewee Model.

Os atributos são objetos herdados das classes do Peewee, eles equivalem aos tipos dos campos do SQL, e podem ser customizados através de seus atributos.

Após isso temos a meta classe (Meta) que ao ser herdada define o método de conexão através do objeto $\mathrm{db}$, isso vincula a tabela ao seu Banco de Dados, equivalente ao USE DATABASE do SQL.

Após as classes terem sido modeladas devemos executar o método create_tables(), ele sincroniza o modelo em Python com o Banco de Dados escolhido, podemos fazer uma analogia com o procedimento de restaurar um backup de um arquivo SQL.

Figura 4 - Sincronizando o arquivo Python com o Banco de Dados

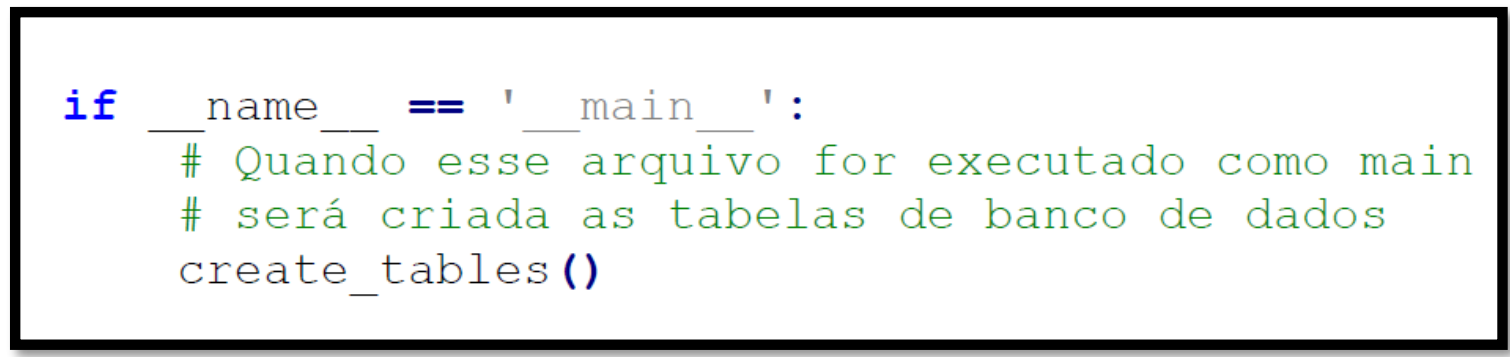

Fonte: SILVA A, COSTA A S e CÂNDIDO D (2019)

Uma maneira comumente adotada para isso é definir o método create_tables() dentro de um if _ name__ = = __ main__', oque garante que ele 
será chamado somente quando o arquivo que o contém seja diretamente executado.

\subsection{POPULANDO O BANCO DE DADOS COM O PEEWEE}

Além de inserirmos os dados diretamente via API, o Peewee também permite que o Banco de Dados seja populado diretamente.

Uma das maneiras de se fazer isso é através do método create das entidades.

Figura 5 - Populando o Banco de Dados com o método create()

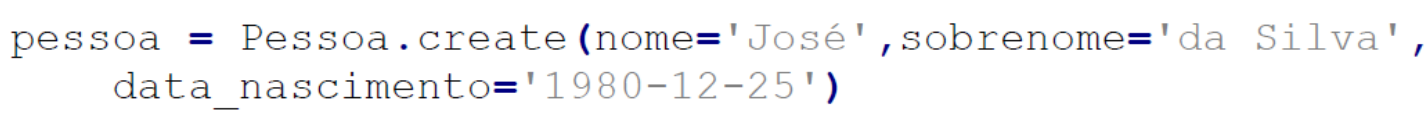

Fonte: SILVA A, COSTA A S e CÂNDIDO D (2019)

Entretanto em diversas ocasiões é necessário inserir uma grande quantidade de dados, para isso o Peewee disponibiliza o método insert_many() que torna mais prático essa tarefa em relação ao método create().

Figura 6 - Populando o Banco de Dados com o método insert_many()

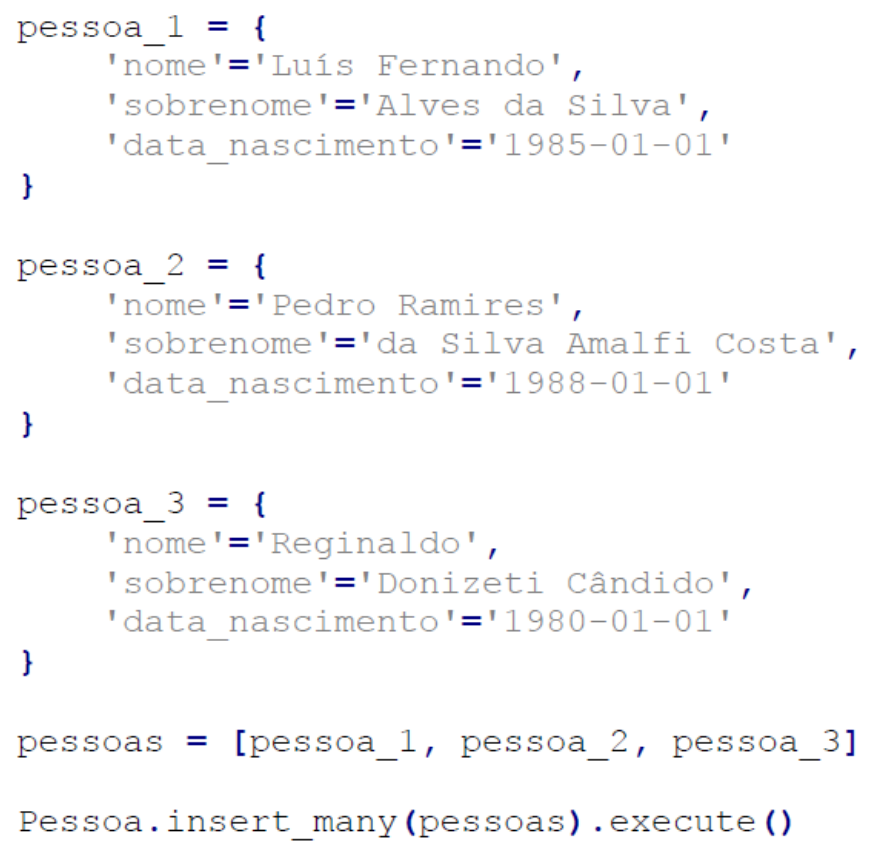

Fonte: SILVA A, COSTA A S e CÂNDIDO D (2019) 
Como visto na imagem anterior primeiramente nos estruturamos as tuplas a serem inseridas na tabela na forma de dicionários Python, em seguida os dicionários são colocados em uma lista e finalmente esta é utilizada como parâmetro do método insert_many().

\subsection{UTILIZANDO OS MÉTODOS CRUD COM O PEEWEE}

O Peewee permite que realizemos a maioria das operações que podem ser realizadas com SQL, entretanto existe um conjunto básico de ações conhecidos como CRUD que são as quatro operações básicas (criação, consulta, atualização e destruição de dados) realizadas por qualquer Banco de Dados.

\subsubsection{CREATE COM O PEEWEE}

A operação Create equivale ao comando INSERT do SQL, o método padrão para criar dados é o create() já apresentado anteriormente, além dele e do também explicado método insert_many(). Também é possível utilizar o método save() equivale ao comando UPDATE do SQL para inserir dados.

Figura 7 - Inserindo dados com o método save()

data $=$ Pessoa()

data.nome = 'Maria'

data. sobrenome $=$ 'Lina Dias'

data.data nascimento = '1921-08-08' data.save ()

Fonte: SILVA A, COSTA A S e CÂNDIDO D (2019) 


\subsubsection{READ COM O PEEWEE}

A operação Read equivale ao comando SELECT do SQL, o método padrão para ler dados é o select(), mas este pode ser combinado com outros métodos para ser fazer diversas filtragem, assim como na linguagem SQL combinamos outras instruções com o SELECT.

Figura 8 - Selecionando dados com o método select ()

\section{data $=$ Pessoa.select ()}

Fonte: SILVA A, COSTA A S e CÂNDIDO D (2019)

\subsubsection{UPDATE COM O PEEWEE}

A operação Update equivale ao comando UPDATE do SQL, os métodos padrões para atualizar dados são save() e update().

Figura 9 - Atualizando dados com o método update()

data $=$ Pessoa update (nome = 'João'). Where(Pessoa.nome = = 'Joao') data.execute()

Fonte: SILVA A, COSTA A S e CÂNDIDO D (2019)

\subsubsection{DELETE COM O PEEWEE}

A operação Delete equivale ao comando DELETE do SQL, os métodos padrões para remover dados são delete_instance() e delete().

Figura 10 - Removendo dados com o método delete() 
data = Pessoa.delete(). where (Pessoa.nome == 'Vitor') data.execute ()

Fonte: SILVA A, COSTA A S e CÂNDIDO D (2019) 


\section{CONCLUSÃO}

Os Banco de Dados são uma parte fundamental da maioria dos aplicativos modernos, entretanto sua modelagem e operação requer conhecimento especializado.

Muitas vezes esse conhecimento não é de domínio de programadores iniciantes, além disso existem diferenças pontuais entre as instruções SQL dos diversos SGBDs.

O uso de softwares ORM nos propicia uma maior facilidade e dinamismo ao se trabalhar com Banco de Dados, pois basta que o programador tenha conhecimento da linguagem de programação e de orientação a objetos para que ele possa ter acesso aos SGBDs.

O Peewee torna essa tarefa fácil, por ser um ORM minimalista ele exige poucas configurações para sua utilização isso aliado com a facilidade de uso da linguagem de programação Python o tornam uma boa escolha para essa tarefa. 


\section{REFERÊNCIAS}

ALVES - Sistema de gerenciamento de banco de dados - URL (https://dicasdeprogramacao.com.br/o-que-e-um-sgbd/) acessado em 18/09/2019 publicado em 01/04/2013.

ALVES GUSTAVO FURTADO DE OLIVEIRA - Você precisa saber o que é SQL - URL (https://dicasdeprogramacao.com.br/o-que-e-sql/) visto em 18/09/2019 publicado em 23/04/2013.

CALLIGARO - Persistência (ciência da computação) - URL (https://blogs.msdn.microsoft.com/windowsmobile/2005/07/14/why-persistentstorage-is-a-good-thing/) acessado em 18/09/2019 publicado em 14/06/2005.

DEVMEDIA - Introdução ao PostgreSQL - URL (https://www.devmedia.com.br/introducao-ao-postgresql/6390) acessado em 18/09/2019.

DEVMEDIA - Mapeamento objeto-relacional - URL (https://www.devmedia.com.br/orm-object-relational-mapper/19056) acessado em 18/09/2019 publicado em 25/01/2011.

DEVMEDIA - ORM: Object Relational Mapper - URL (https://www.devmedia.com.br/orm-object-relational-mapper/19056) acessado em 18/09/2019.

OLIVEIRA - SQLite - URL (https://terminalroot.com.br/2011/12/exemplode-utilizacao-de-sqlite-com-php.html) acessado em 18/09/2019 publicado em $11 / 012 / 2011$.

PEEWEE - API Documentation - URL (http://docs.peeweeorm.com/en/latest/peewee/api.html) acessado em 18/09/2019.

PEEWEE - peewee - URL (http://docs.peewee-orm.com/en/latest/) acessado em 18/09/2019.

PEEWEE - Querying - URL (http://docs.peeweeorm.com/en/latest/peewee/querying.html) acessado em 18/09/2019. 
PEEWEE - Quickstart - URL (http://docs.peeweeorm.com/en/latest/peewee/quickstart.html) acessado em 18/09/2019.

PROFESSOR DIGITAL - Normalização de dados e as formas normais URL (https://www.luis.blog.br/normalizacao-de-dados-e-as-formasnormais.html) acessado em 18/09/2019.

PROFISSAODBA - Evolução dos Bancos de Dados e SGBDs - URL (https://profissaodba.wordpress.com/historico-dos-bancos-de-dados/) acessado em 18/09/2019.

STUTTGART MICHELL - Peewee: Um ORM Python minimalista - URL (http://pythonclub.com.br/peewee-um-orm-python-minimalista.html) visto em 18/09/2019 publicado em 20/07/2017.

ZANZARINI

MySQL

URL

(https://www.brasilwebhost.com.br/blog/mysql-o-que-e-e-qual-a-suaimportancia/) acessado em 18/09/2019. 